\title{
Congenital Deafness with Ear Deformities in Arthrogryposis Multiplex Congenita (AMC)
}

\author{
Ryan Chin Taw Cheong ${ }^{1}$, Bhavesh Patel ${ }^{1}$, Bik Kotecha ${ }^{1,2}$, Gaurav Kumar ${ }^{1}$ \\ ${ }^{1}$ Barking, Havering and Redbridge University Hospitals NHS Trust, ${ }^{2}$ Royal National \\ Throat, Nose and Ear Hospital
}

\section{Introduction}

First described by Otto in 1841, arthrogryposis multiplex congenital $(\mathrm{AMC})$ is a rare disorder currently listed under the National Organisation of Rare Disorders (1). It is characterized by joint contractures that are present at birth in at least two areas of the body. The authors report an uncommon case of associated congenital deafness with ear deformity in a patient with AMC.

\section{Methods}

54 year old female patient with bilateral congenital profound mixed hearing loss requiring hearing aids from birth was reviewed with the otoendoscope revealing a number of otological deformities including low set ears, deformed pinna, deformed external auditory canal and possible middle ear deformity. CT-temporal bones revealed upslanting external auditory canals but no overt deformity of the ossicles. The patient was managed conservatively after exclusion of evidence of cholesteatoma on radiological imaging.

Right ear
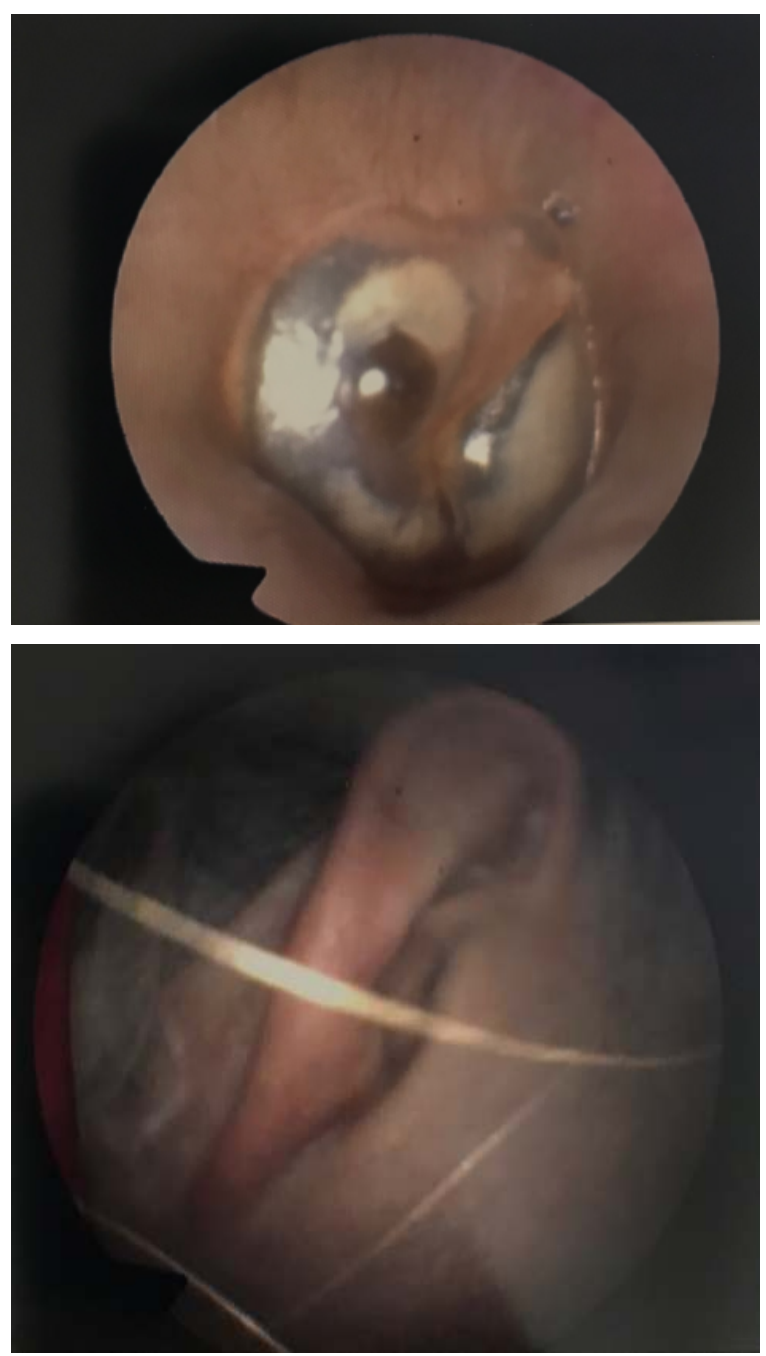

Left ear

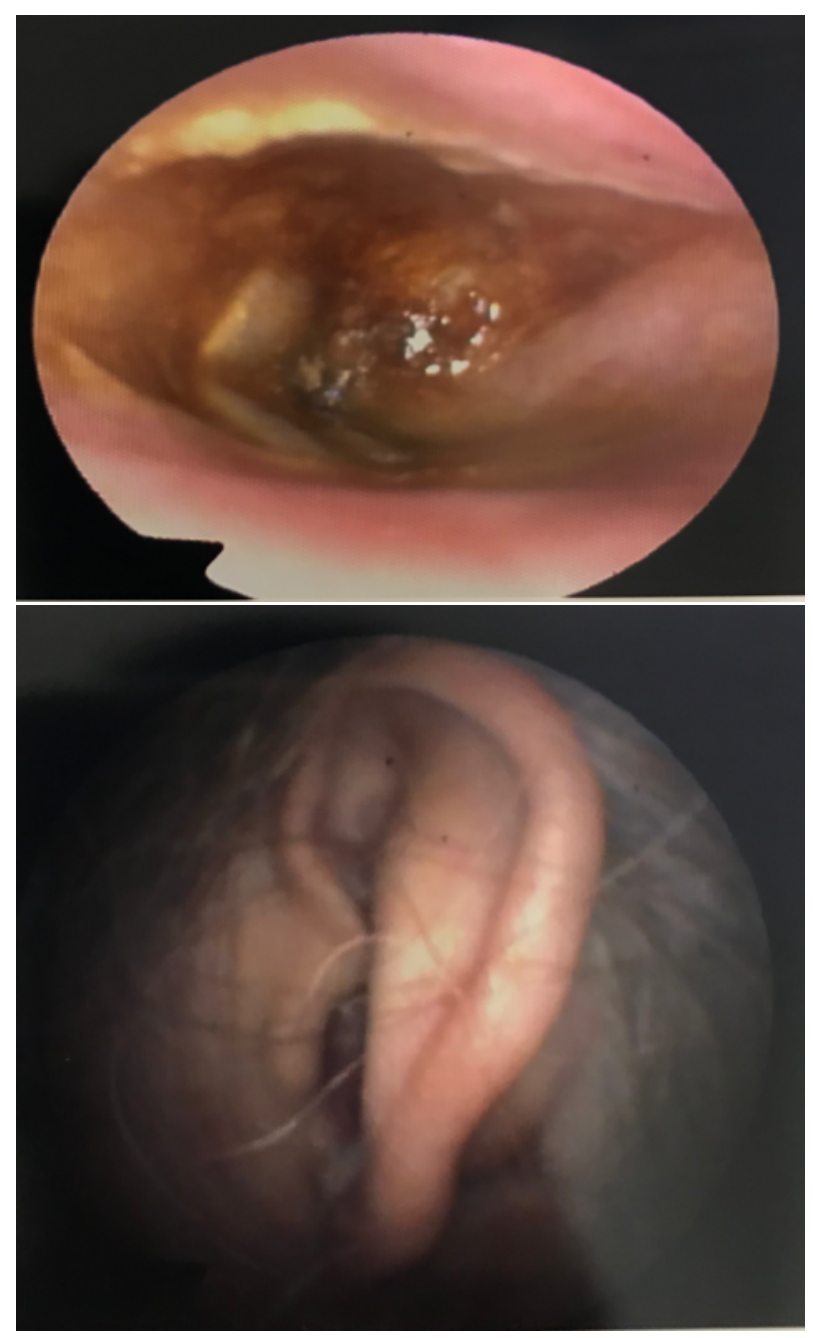

\section{Results}

Only one other case with associated middle ear deformity in AMC has been reported in the literature (2).
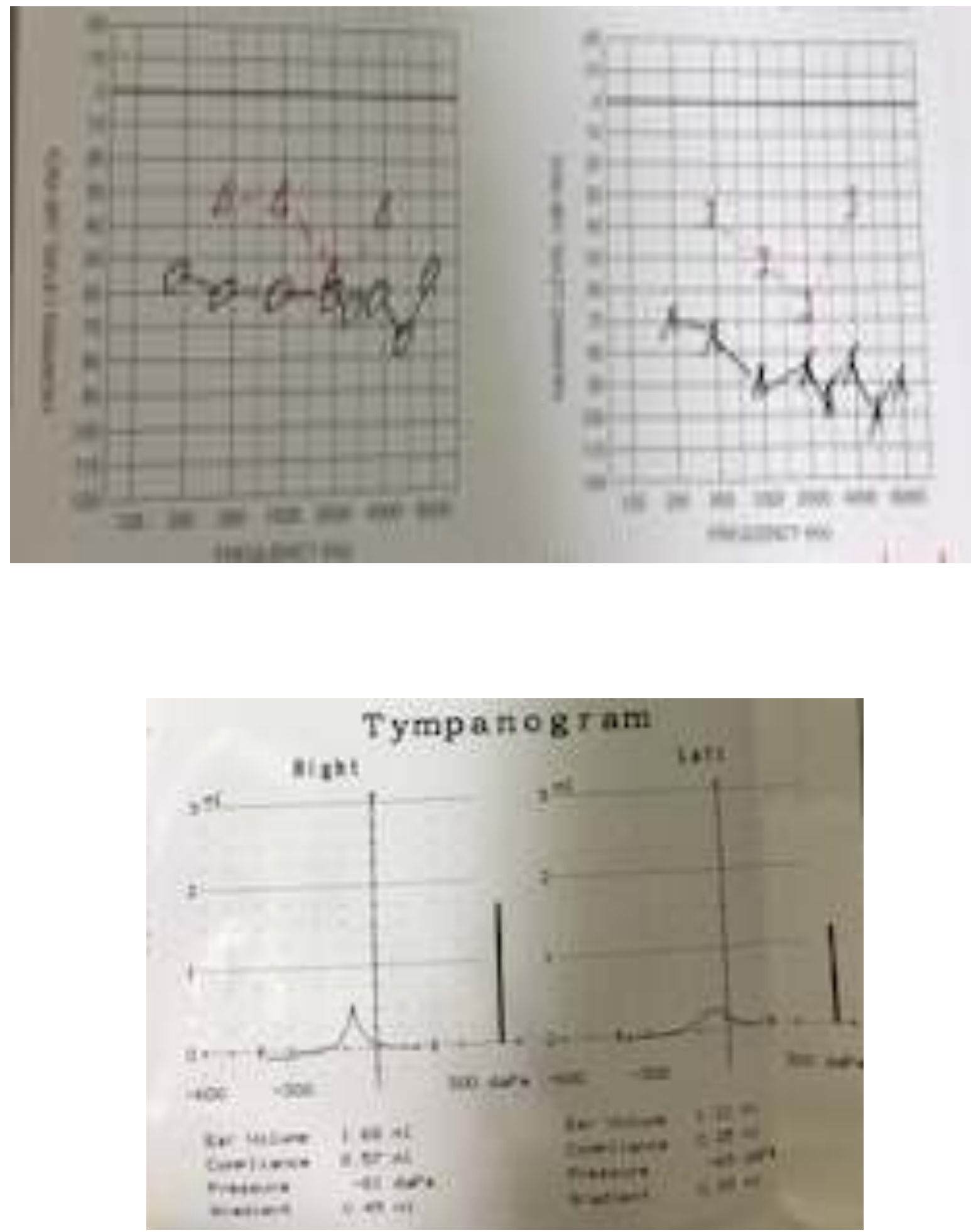

\section{Conclusion}

Associated congenital deafness and ear deformities are possible features in patients with AMC that the otorhinolaryngologist should include in their clinical repertoire.
References

1. Otto AW, Peltier LF. A Human Monster with Inwardly Curved Extremities. Clinical Orthopaedics and Related Research. 1985 Apr 1;194:4-5.
2. Quinn SJ, Bleach NR, Richards AE. Middle ear deformity in arthrogryposis multiplex congenita. Annals of Otology, Rhinology \& Laryngology. 1994 Sep; 103(9):729-31. 